Nonlin. Processes Geophys., 15, 557-565, 2008

www.nonlin-processes-geophys.net/15/557/2008/

(C) Author(s) 2008. This work is distributed under

the Creative Commons Attribution 3.0 License.

\title{
Extreme event return times in long-term memory processes near $1 / f$
}

\author{
R. Blender, K. Fraedrich, and F. Sienz \\ Universität Hamburg, Meteorologisches Institut, Bundesstrasse 55, 20146 Hamburg, Germany \\ Received: 2 August 2007 - Revised: 17 June 2008 - Accepted: 17 June 2008 - Published: 16 July 2008
}

\begin{abstract}
The distribution of extreme event return times and their correlations are analyzed in observed and simulated long-term memory (LTM) time series with $1 / f$ power spectra. The analysis is based on tropical temperature and mixing ratio (specific humidity) time series from TOGA COARE with 1 min resolution and an approximate $1 / f$ power spectrum. Extreme events are determined by PeakOver-Threshold (POT) crossing. The Weibull distribution represents a reasonable fit to the return time distributions while the power-law predicted by the stretched exponential for $1 / f$ deviates considerably.

For a comparison and an analysis of the return time predictability, a very long simulated time series with an approximate $1 / f$ spectrum is produced by a fractionally differenced (FD) process. This simulated data confirms the Weibull distribution (a power law can be excluded). The return time sequences show distinctly weaker long-term correlations than the original time series (correlation exponent $\bar{\gamma} \approx 0.56$ ).
\end{abstract}

\section{Introduction}

Long-term memory (LTM) is a ubiquitous phenomenon in natural time series and mainly identified by power-laws characterized by a single correlation exponent $\gamma$ in the correlation function, $C(t) \sim t^{-\gamma}$ (Fraedrich and Blender, 2003). In many observed time series, predominantly sea surface temperatures, $1 / f$ power spectra are found related to small $\gamma$ (Weissman, 1988; Monetti et al., 2003). In the current discussion on anthropogenic climate change, the simulation of LTM becomes relevant since anthropogenic trends may be masked by low frequency internal variability (Blender and Fraedrich, 2003).
Even weak LTM (with $\gamma$ slightly below 1) has considerable impacts on return times of extreme events (Altmann and Kantz, 2005; Eichner et al., 2007). An obvious reason for this effect is the clustering of threshold crossings during periods with high averages (Bunde et al., 2005). The distribution of return times $t_{r}$ in the presence of LTM is approximately given by a stretched exponential, $p \sim \exp \left(-t_{r}^{\gamma}\right)$, where the exponent is assumed to be identical to the correlation exponent $\gamma$. The stretched exponential is motivated by the study of Newell and Rosenblatt (1962) who derived an upper bound for the probability of no zero crossings in power-law correlated Gaussian processes. Olla (2007) applied an $\epsilon$-expansion for $\gamma=1-\epsilon$ and obtained a stretched exponential distribution with exponent $\gamma$. Stretched exponential distributions are found for linear systems with LTM (Altmann and Kantz, 2005). There are classes of nonlinear dynamical systems which show algebraic (power-law) distributions (Zaslavsky, 2002). For inter-event distributions of earth quakes Corral (2004) suggests a gamma distribution. The long-term memory does not only alter the distribution of return times but also their temporal correlations which are the basis for the return time predictability (Bunde et al., 2004; Altmann and Kantz, 2005).

The present study is motivated by the abundance of observed nonstationary $1 / f$ time series which are at the border of stationarity. The aim of this paper is to analyse extreme event return time statistics in high resolution observations of tropical boundary layer temperature and humidity which both reveal a $1 / f$-spectrum. The results are compared with a simulated time series obtained by a long simulation of a stationary fractionally differenced process (FD) with a power spectrum in the vicinity of the $1 / f$. To evaluate potential predictability, long-term correlations of return times in this time series are estimated.

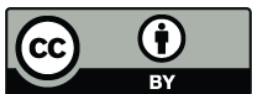

Correspondence to: R. Blender

(richard.blender@zmaw.de)

Published by Copernicus Publications on behalf of the European Geosciences Union and the American Geophysical Union. 
The paper is organized as follows: In Sect. 2 LTM is defined and available results on return time distributions are summarized. The long term memory properties and the return time distributions of the observational data are determined in Sect. 3. In Sect. 4 simulated time series are compared and the correlation properties of the extreme event intervals are analyzed. The Sect. 5 concludes with a summary and discussion.

\section{Estimating long-term memory and extreme event re- turn time statistics}

For the estimation of long-term memory (LTM, Beran, 1994) several methods are available. We compare results of the Detrended Fluctuation Analysis (DFA, Peng et al., 1994) with fits of FARIMA $(p, d, 0)$ processes (Hosking, 1981). The FARIMA processes are able to assess the contributions of short- and long-term components. The distribution of the extreme event return times is altered in the presence of LTM since long periods with anomalous low or high persistent deviations occur. The correlations between successive extreme event return times are useful for the prediction of extreme event return times.

\subsection{Long-term memory analysis}

A time series has long-term memory (LTM, also denoted as long-term persistence) if the correlation function $C(t)$ is not integrable (Beran, 1994). For a long-term power-law decay, $C(t) \sim t^{-\gamma}$, LTM is equivalent to $\gamma>0$. Empirical time series have LTM if the autocorrelation follows a power-law with exponent $0<\gamma<1$ for the largest time scales present. LTM is ubiquitous in nature and shows up mainly in temperature records (Fraedrich and Blender, 2003; Huybers and Curry, 2006). The exponent $\beta$ of the power spectrum, $S(f) \sim f^{-\beta}$, and the correlation exponent are related by $\beta=1-\gamma$, hence the power spectrum increases with decreasing frequency for $\gamma<1$.

To determine LTM properties two methods are applied (see Sect. 2.1): Detrended fluctuation analysis (DFA, Peng et al., 1994), and an estimation of the parameters in FARIMA $(p, d, 0)$ processes (Hosking, 1981).

The two methods are independent complements for the analysis of our data and inhibit an erroneous detection of LTM: While there is a known LTM detection problem in the DFA in short term memory time series (Maraun et al., 2004), this method does not require any model assumption (for example normality of the data). The FARIMA process is ideal for the detection of short- and long-term memory, in addition, it allows a significance test for the number of parameters, however, normality of the data is required. (i) The DFA determines fluctuations $F(\tau)$ on time scales $\tau$ in stationary anomaly sequences with LTM. Trends in the time series can be eliminated by extensions of the DFA (Fraedrich and Blender, 2003).

(ii) To assess the contributions of short- and long term memory components, fits of autoregressive processes (AR) and fractionally integrated autoregressive process are considered. In the following, FAR is used as a short notation for FARIMA $(p, d, 0)$ (Hosking, 1981) which includes an autoregressive (AR) process of order $p$ and a fractionally differenced (FD) process with dimension $d$.

The AR process is defined by

$\phi(B) x_{t}=\epsilon_{t}$

where $B$ is the backshift operator defined by $B x_{t}=x_{t-1}$, and $\epsilon_{t}$ is white noise. Using the coefficients $a_{n}$, the polynomial $\phi(B)$ is

$\phi(B) x_{t}=x_{t}-\sum_{n=1}^{p} a_{n} x_{t-n}$

The FD process (Hosking, 1981) is derived from

$(1-B)^{d} x_{t}=\epsilon_{t}$

and leads to an AR process of infinite order

$x_{t}=\sum_{n=1}^{\infty} a_{n} x_{t-n}+\epsilon_{t}, \quad a_{n}=-\frac{\Gamma(n-d)}{\Gamma(-d) \Gamma(n+1)}$

For low frequencies the FD process shows a scaling power spectrum $S(f) \sim f^{-\beta}$ with spectral exponent $\beta=2 d$ and correlation exponent $\gamma=1-2 d$.

The FAR process is given by the combination

$\phi(B)(1-B)^{d} x_{t}=\epsilon_{t}$

and is determined by $p$ coefficients in the AR and the dimension $d$.

\subsection{Extreme event return distributions}

An extreme event in a time series $x_{i}, i=1, \ldots, N$, crosses a given threshold $q$ with $x_{i}>q$. The return time $t_{r}$ between two extreme events is the time interval between two events with $x_{i}>q$ and $x_{i+t_{r}}>q$ and lower values $x_{j}<q$ in between, $i<j<i+t_{r}$. The mean return time $R_{q}$ depends on the threshold $q$ and is approximated by the probability distribution function (pdf) $D(x)$ of the time series

$R_{q}^{-1}=\int_{q}^{\infty} D(x) d x$

In the present paper, the threshold $q$ is determined to obtain a specific value of $R_{q}$. For uncorrelated data, the return times are exponentially distributed following a Poisson process

$p_{q}\left(t_{r}\right)=\frac{1}{R_{q}} \exp \left(-t_{r} / R_{q}\right)$ 
LTM leads to periods with anomalous persistent low or high deviations. During such periods extreme high values are either rare (for low anomalies) or frequent (during high anomalies). Thus return time statistics shows clustering which is not observed in time series without memory.

For LTM time series stretched exponential return time distributions are suggested (Bunde et al., 2004; Altmann and Kantz, 2005; Eichner et al., 2007)

$p_{q}\left(t_{r}\right) \approx \frac{a_{\gamma}}{R_{q}} \exp \left[-\left(b_{\gamma} t_{r} / R_{q}\right)^{\gamma}\right]$

Note that the scaling exponent $\gamma$ is conjectured to be equal to the correlation exponent which characterizes LTM. The coefficients $a_{\gamma}=\gamma \Gamma(2 / \gamma) / \Gamma^{2}(1 / \gamma)$ and $b_{\gamma}=\Gamma(2 / \gamma) / \Gamma(1 / \gamma)$ are determined by normalisation of $p_{q}$ and the condition for the mean, $R_{q}=<t_{r}>$; $\Gamma$ is the gamma-function.

In the limit $\gamma \rightarrow 0$, the stretched exponential approaches a power law

$\log p_{q}\left(t_{r}\right) \sim-s \log t_{r}+$ const

with the exponent

$s=\lim _{\gamma \rightarrow 0} \gamma b_{\gamma}^{\gamma}=1.5$

Altmann and Kantz (2005) and Eichner et al. (2007) consider the correlation exponents $0.05<\gamma<1$ that is, between almost $1 / f$ and white noise. Eichner et al. (2007) show that the stretched exponential is valid for several types of distributions $D$.

For small return times, $t_{r} \ll R_{q}$, the observed distribution deviates from the stretched exponential (8) and scales as (see Eq. (10) in Eichner et al., 2007).

$R_{q} p_{q}\left(t_{r}\right) \sim\left(\frac{t_{r}}{R_{q}}\right)^{s^{\prime}}$

with the proposed value $s^{\prime}=\gamma^{\prime}-1, \gamma^{\prime} \approx \gamma$ for Gaussian density. For large return times $\left(t_{r} \gg R_{q}\right)$ the limit of the distribution (8) is

$R_{q} p_{q}\left(t_{r}\right) \sim \exp \left[-\left(b t_{r} / R_{q}\right)^{\gamma}\right]$

The stretched exponential is accepted as an approximate representation for linear LTM processes (Altmann and Kantz, 2005; Eichner et al., 2007).

An alternative to the stretched exponential distribution (8) is the Weibull distribution (Sornette, 2006; Abaimov et al., 2007) with the scale parameter $\tau$ and the shape parameter $\gamma$

$p_{W}\left(t_{r}\right)=\frac{\gamma}{\tau}\left(\frac{t_{r}}{\tau}\right)^{\gamma-1} \exp \left[-\left(t_{r} / \tau\right)^{\gamma}\right]$

Note that the Weibull distribution for $\gamma<1$ is frequently denoted stretched exponential distribution; this, however, differs from (8) by the prefactor $\sim t^{\gamma-1}$. Without reference to Weibull, the power-law (11) is suggested by Eichner et al. (2007) in their Eq. (10) to correct the pure stretched exponential, $\sim \exp \left(-t^{\gamma}\right)$, for small return times.

The advantages of the Weibull distribution for the characterization of extreme event return times are:

(i) The Weibull distribution (13) combines (8) and the short time limit (11) and describes the observed distribution in a wide range of return times.

(ii) The cumulative distribution function is known, $F_{W}\left(t_{r}\right)=1-\exp \left[-\left(t_{r} / \tau\right)^{\gamma}\right]$, and the mean recurrence time is determined by $R=\tau \Gamma(1+1 / \gamma)$. This cumulative distribution function is useful for statistical analyses.

(iii) According to Sornette (2006) power-laws can be approximated by the Weibull distribution in arbitrary intervals to any prescribed accuracy.

The fit of the discrete power-law and Weibull distribution to the return time series is performed following Clausset et al. (2007). The approach fits the parameters of the distributions (exponents for power-laws; shape and scale parameters for Weibull) using Maximum Likelihood estimation and determines an optimal range (restricted by a minimum return time cutoff) by minimizing the Kolmogorov-Smirnov distance.

The correlations between successive extreme event return times are one of the most useful aspects in practical applications of extreme value theory. Given time series with weak LTM (correlation exponents $\gamma=0.4$ and 0.7), Bunde et al. (2004) analyse the respective return times arranged in a sequence, and find their long-term correlation exponents $\gamma$ to be similar to the exponents of the original time series. It is expected that this relationship changes distinctly for very strong LTM due to its close vicinity to the nonstationarity threshold $1 / f$.

In this paper, extreme events are determined by the PeakOver-Threshold (POT) method with different thresholds $q$, which are adjusted for mean return times $R_{q}$. The detrended fluctuation analysis (DFA) is employed to determine the LTM of the observational data and the recurrence times in the simulated data. Fits of FARIMA process support the LTM analysis of observational data. The time series are simulated by fractionally differenced processes (FD, Hosking, 1981). The fits of the power-law and the Weibull distributions are performed by the code available from Clausset et al. (2007). For all other calculations we use the statistics software R (R Development Core Team, 2005).

\section{High resolution observational data}

The observed time series analyzed in this study are obtained during the TOGA-COARE experiment (November 1992-February 1993, Data Processing Center/Data Archive and Distribution Center for COARE Surface Meteorological Data, Florida State University, COARE-MET; Webster 
and Lukas, 1992). The aim of the international field experiment TOGA COARE during 1992-1993 was to study the atmospheric and oceanic processes over the western $\mathrm{Pa}$ cific. The data measured at the Research Vessel (R/V) Kexue $\left(3.9^{\circ} \mathrm{S}, 155.9^{\circ} \mathrm{E}\right)$ encompasses boundary layer near surface air temperature and the mixing ratio with one minute resolution (Fig. 1); this data set has been corrected by Lucas and Zipser (2000). In the air temperature time series the diurnal cycle (daily mean with 1 min resolution) is removed for the analysis. The weak diurnal cycle in the mixing ratio is not removed since this does not change the result.

The mixing ratio (Fig. 1c) reveals the presence of a large scale event during the first part of the time series (due to a passing 40-day wave). The fluctuations of the temperature and the mixing ratio are characterized by a $1 / f$ power spectrum in a wide range of time scales (Yano et al., 2001, 2004). Only a part of this time series $\left(8.8 \cdot 10^{4}\right.$ time steps, roughly 61 days) is analyzed to keep the number of missing values below $<5 \%$. The missing values are replaced by the mean and no attempt has been made to determine the effect of these replacements.

The overall behaviour of the data indicates nonstationarities in both time series. The frequency distributions for both time series (Fig. 1b, d) show deviations from Gaussian, which are, however, not substantial and presumably related to the nonstationarity.

\subsection{Long term memory analysis}

To determine LTM properties of the two observed time series two methods are applied (see Sect. 2.1): Detrended fluctuation analysis (DFA, Peng et al., 1994), and an estimation of the parameters in FARIMA $(p, d, 0)$ process (Hosking, 1981).

(i) The DFA spectra in Fig. 2a, b show scaling fluctuation spectra, $F(\tau) \sim \tau^{\alpha}$, with exponents $\alpha \approx 1 \ldots 1.1$ close to a $1 / f$-spectrum $(\alpha=1)$ for the temperature and the mixing ratio. The power spectrum is closely related to $F(\tau)$ and scales as $S(f) \sim f^{-\beta}$ with exponents $\beta=2 \alpha-1 \approx 1 \ldots 1.2$; the correlation exponents are $\gamma=1-\beta \approx 0 \ldots-0.2$. Note that the temperature fluctuation spectrum in Fig. 2a approaches $\alpha \approx 1(\gamma=0)$ for long time periods $\left(t_{r}>10^{3} \mathrm{~min}\right)$ during two decades. An analysis of a trend-eliminating version of the DFA yields the same exponents.

(ii) Short- and long term memory contributions are assessed by fits of autoregressive processes (AR) and fractionally integrated autoregressive process (FAR); see Sect. 2.1. To determine the optimal number of parameters in the FAR fit, the Akaike information criterion (AIC) is used based on the minimum of

$$
\mathrm{AIC}=-2 \log (\mathcal{L})+2 k
$$

where $\mathcal{L}$ is the maximized likelihood function and $k$ the number of estimated parameters. For temperature and mixing ratio it appears that the FAR process is superior to AR processes for small numbers of coefficients (Fig. 3a,b). The mixing ratio shows a higher preference for the FAR than temperature, which can be explained by the higher degree of scaling (Fig. 2b). Furthermore, a maximum likelihood ratio test ( $99 \%$ significance) supports a lower degree of the autoregressive component in the FAR for the mixing ratio.

Likelihood ratio tests are performed to test whether higher order models give significant improvement compared to lower order models. FAR-models are tested against all (AR and FAR) lower order models, while the test for the ARmodels is only performed for lower order. Filled symbols (Fig. 3) show significance against lower order models on the 99\% significance level.

For both observed datasets FAR-models outperform the ARs for all model orders below $p=7$ according to the Akaike information criterion and the likelihood ratio test. Even for temperature (Fig. 3a), where the information criterium looks quite similar for higher model orders, the likelihood ratio test prefers the FAR-models. However, the likelihood ratio test for temperature does not indicate an optimal model order. The mixing ratio (Fig. 3b) can best be characterized by an FAR-model including four additional AR coefficients with the correlation exponent $\gamma \approx 5 \cdot 10^{-4}$. Since the model of choice is less clear for the temperature we consider the correlation exponents and their standard deviations. The correlation exponents decay from $\gamma=0.016$ for $p=0$ to $\gamma \approx 10^{-4}$ for $p \geq 2$; the standard deviations are below $\approx 2 \cdot 10^{-3}$. In summarising we conclude that the spectra of both observed time series can be considered as $1 / f$.

\subsection{Return time distributions}

The return time distributions $p_{q}\left(t_{r}\right)$ for temperature and mixing ratio are determined for the mean return time $R_{q}=100$ (6). For the data the complementary cumulative distribution function (CCDF) is determined, $C_{F}\left(t_{r}\right)=1-F\left(t_{r}\right)$, where $F\left(t_{r}\right)$ is the cumulative distribution function. Scaling of the distribution function is preserved in the CCDF with an exponent reduced by 1 . Unfortunately, a fit of the stretched exponential distribution is inhibited by insurmountable numerical difficulties.

The distributions in Fig. 4a, b are compared with the fits of Weibull distributions (13) and power-laws. The Weibull parameters, the power-law exponents, and the cutoffs are given in Table 1 together with confidence intervals, which are determined by resampling with replacement (1000 samples). For temperature and mixing ratio the powerlaw exponent $s=1.5$ lies outside the confidence intervalls. 

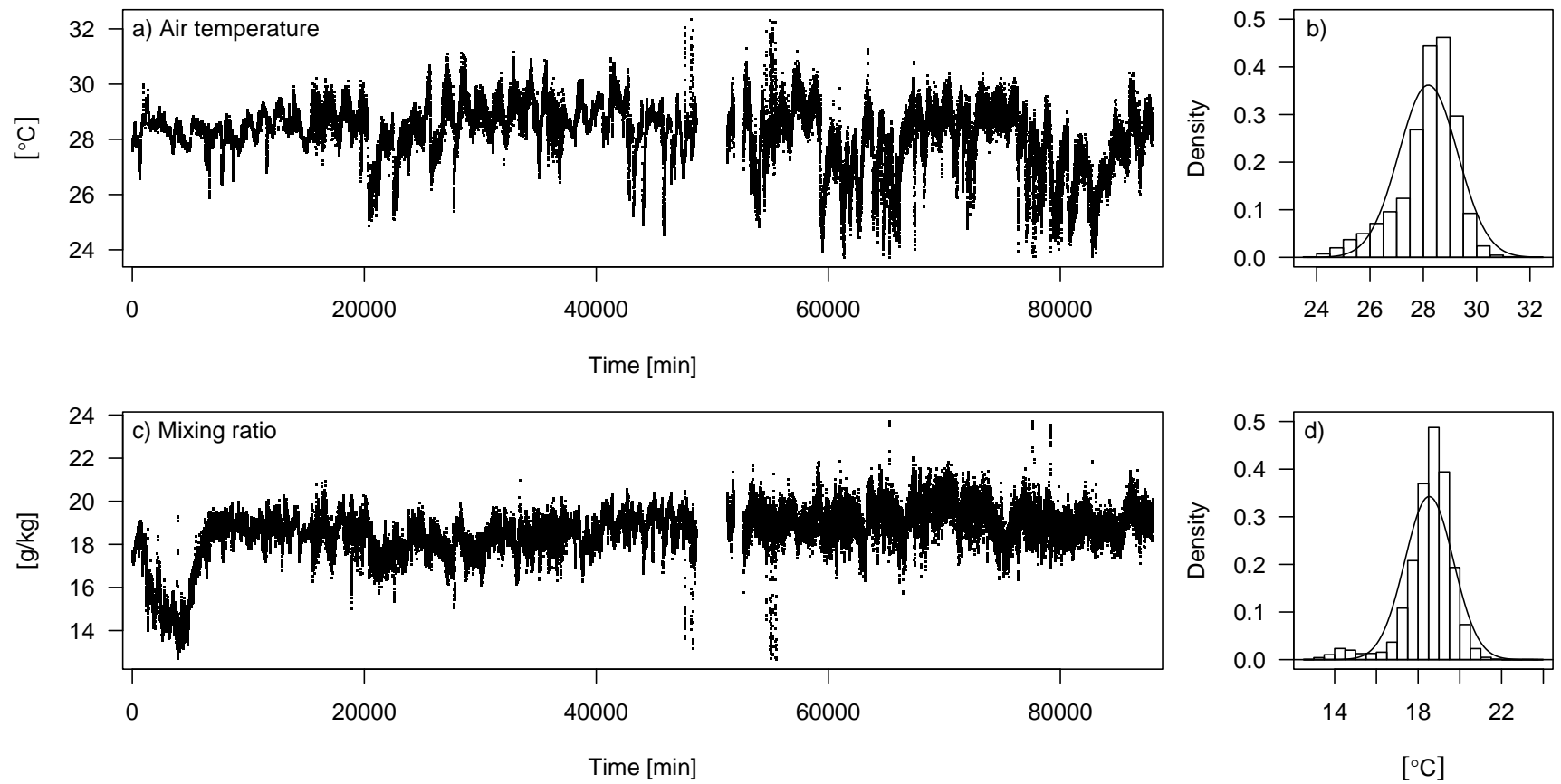

Fig. 1. Observations of (a) atmospheric near surface temperature and and (c) mixing ratio; corresponding frequency distributions in (b) and (d).
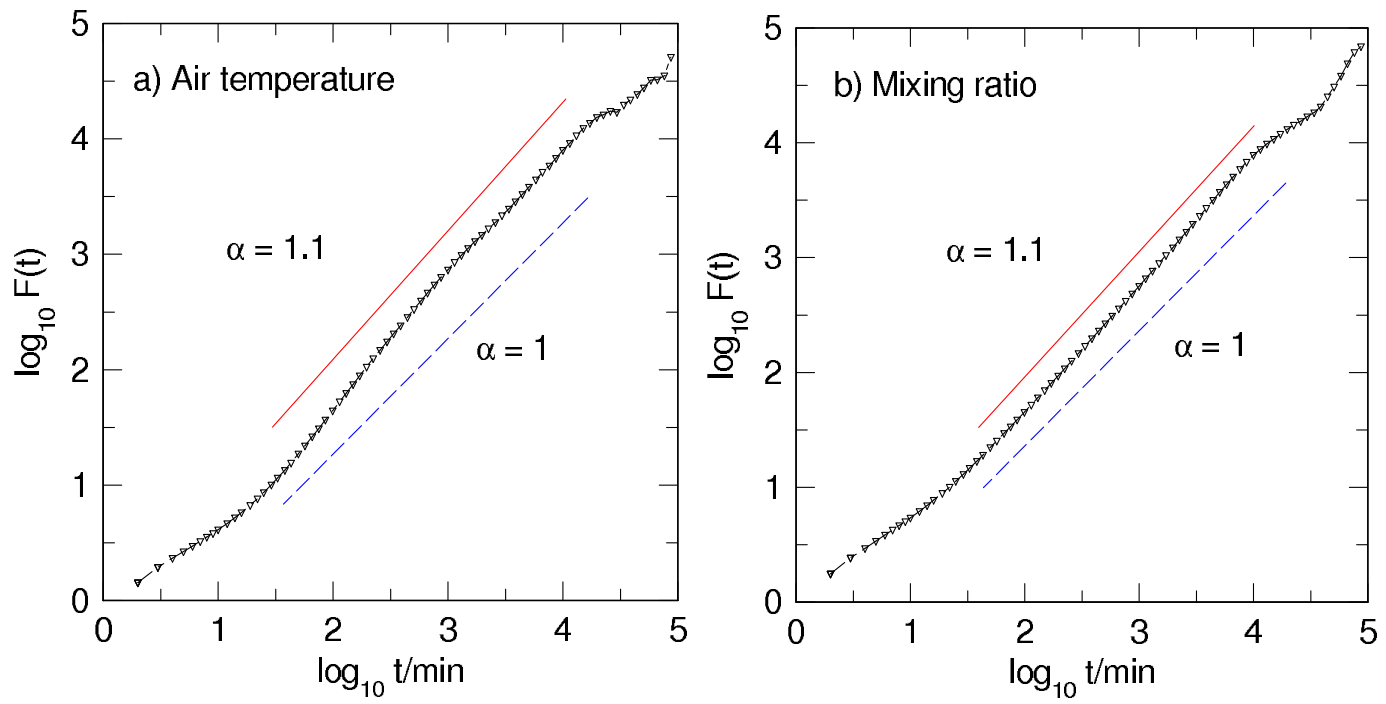

Fig. 2. DFA fluctuation function of (a) atmospheric near surface temperature and (b) mixing ratio at R/V Kexue. The solid (red) lines indicates $\alpha=1.1$, the dashed (blue) lines represents a $1 / f-\operatorname{spectrum}(\alpha=1)$.

The cutoffs are determined by minimizing the Kolmogorov-Smirnov test statistic. The power-law fits are compared with the power-law $s=1.5$ predicted by the limit of the stretched exponential for $1 / f$ noise $(9,10)$. The return time distributions for the two observed time series are reasonably well approximated by Weibull distributions in a wide range of return times. Note that the power-law fits are restricted to narrow ranges (in particular for the mixing ratio) and are obviously worse approximations for the observed distributions. The power-law exponents for air temperature 

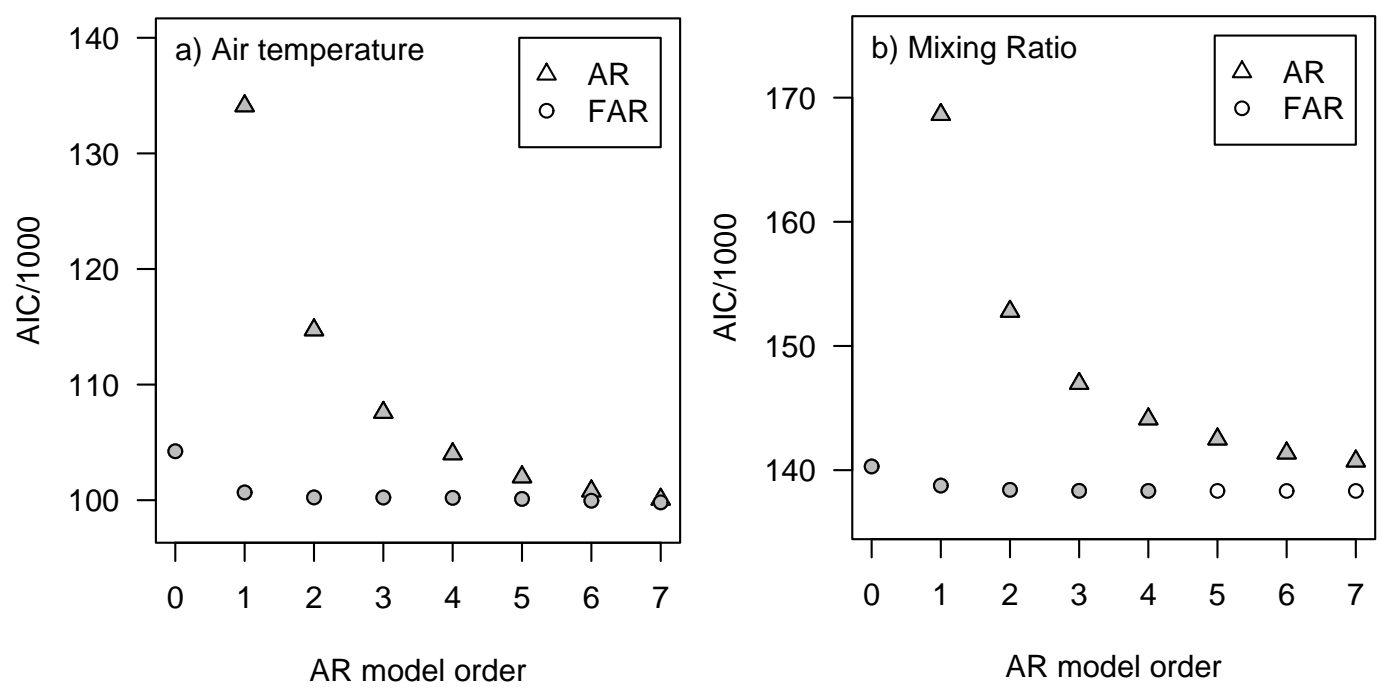

Fig. 3. Akaike Information Criterion (AIC) for AR $(\triangle)$ and FAR (o) models: (a) air temperature and (b) mixing ratio. Filled symbols show significance against lower order models according to likelihood-ratio tests.
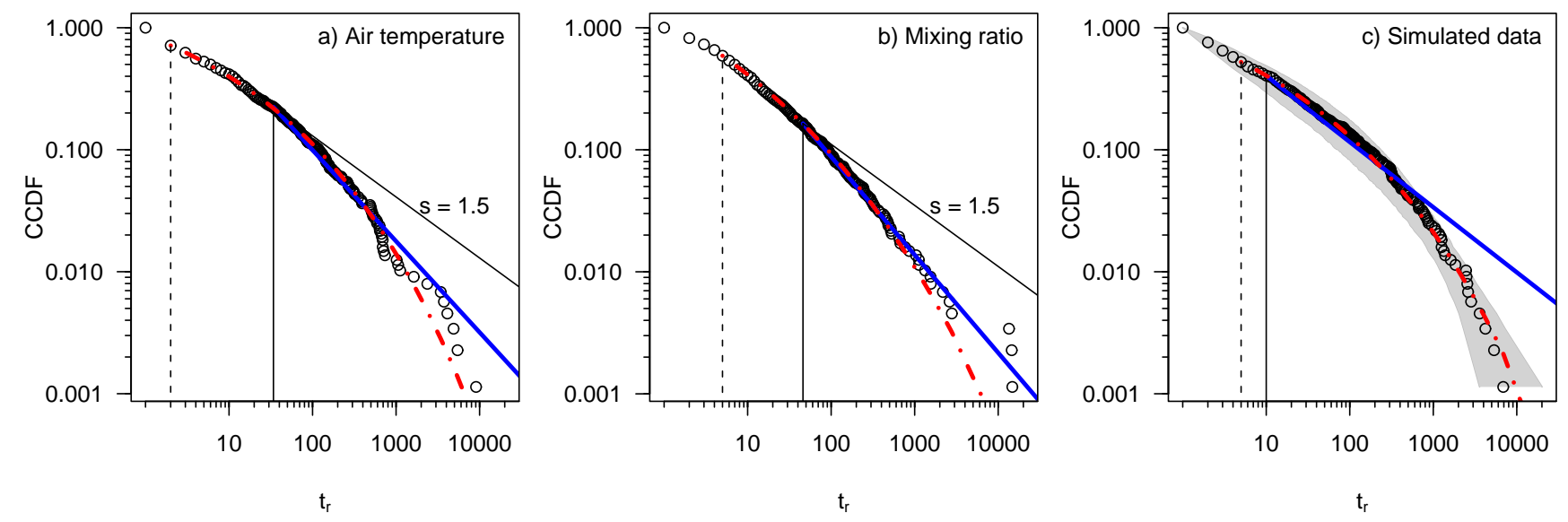

Fig. 4. Complementary cumulative distribution functions for the return times in (a) air temperature, (b) mixing ratio, and (c) $1 / f$ simulated data. Dashed (red) curves denote Weibull distributions (13) and solid (blue) power-laws distributions. Vertical lines denote cutoffs $k_{\text {min }}$. The solid black lines denote the exponent 1.5 for the same cutoffs as the fits (this overlaps with the blue line in (c)). In (c) the $95 \%$ confidence interval is gray shaded.

$(s=1.74)$ and mixing ratio $(s=1.8)$ differ substantially from 1.5 , since this value is beyond the confidence intervals (Table 1).

\section{Simulated data}

Simulated time series with self-similar LTM are generated by a linear autoregressive process. As the AR part (2) is responsible for short memory, the simulated data is simulated by an FD process (4), see Fig. 5 for the time series and the Gaussian frequency distribution. The power spectrum exponent is chosen as $\beta=0.99(d=\beta / 2=0.495, \gamma=0.01)$ to obtain an approximation for a stationary time series with a $1 / f$ power spectrum. To inhibit the impact of finite size effects in the comparison with observational data, the total length of the simulated time series is identical with that of the observed data $\left(N=8.8 \cdot 10^{4}\right)$. For the following analysis of the LTM in the return time sequence, however, a very long time series of $N=10^{8}$ is simulated. 

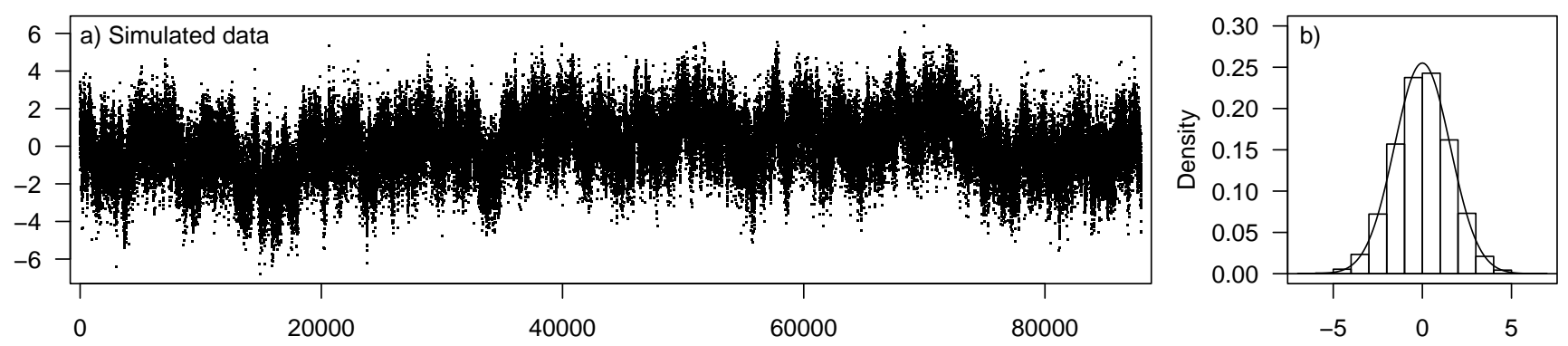

Fig. 5. (a) Simulated data (FD with $\gamma=0.01$ ) and (b) frequency distribution.

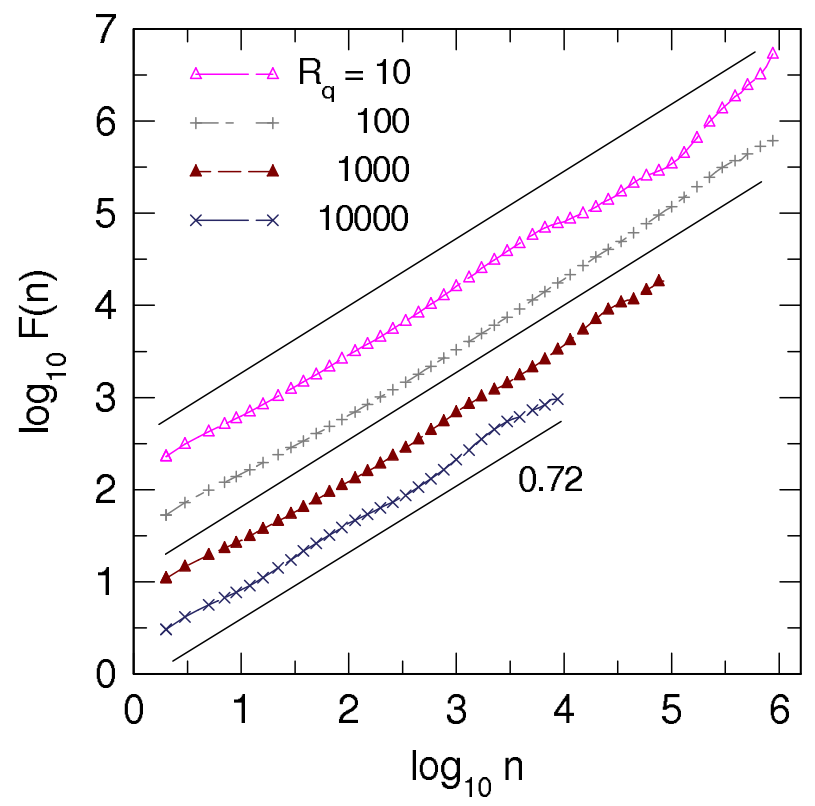

Fig. 6. DFA fluctuation functions for the sequence of return times obtained with a FD $(\gamma=0.01)$ and different thresholds corresponding to the mean return times $R_{q}$ as indicated. $n$ enumerates the return time sequence. The solid lines show the LTM exponent $\alpha=0.72$.

\subsection{Return time distributions}

The distribution $p_{q}\left(t_{r}\right)$ for the return times $t_{r}$ is determined for a threshold $q$ related to a mean return time $R_{q}=100$. The analysis is analogous to Sect. 3.2. The distribution (Fig. 4c) is well approximated by a Weibull distribution. The $95 \%$ confidence interval (gray shaded) is determined by creating 1000 time series with the same parameters. The shape and scale parameters are $\gamma=0.2$ and $\tau=0.29$, respectively. The validity of the power law fit, $p_{q} \sim t_{r}^{-s}$ with the exponent $s=1.53$, is restricted to $t_{r} \approx 10 \ldots .500$. The deviation from $s=1.5$, which is the $1 / f$ limit of the stretched exponential, might originate in either:
Table 1. Values of: estimated parameters for Weibull and powerlaw distributions (with 95\% confidence intervals), and cutoffs for the Weibull (power-law) distribution.

\begin{tabular}{llll}
\hline & temperature & mixing ratio & simulated data \\
\hline shape $\gamma$ & $0.21(0.17,0.27)$ & $0.15(0.11,1.88)$ & $0.19(0.15,0.25)$ \\
scale $\tau$ & $0.36(0.04,1.94)$ & $0.003\left(10^{-5}, 0.04\right)$ & $0.29(0.01,2.68)$ \\
exponent $s$ & $1.74(1.66,1.85)$ & $1.80(1.69,1.94)$ & $1.53(1.49,1.57)$ \\
cutoff $k_{\min }$ & $4(8)$ & $5(46)$ & $5(10)$ \\
\hline
\end{tabular}

(i) The conjecture that the stretched exponential exponent (see Eq. 8) is identical to the correlation exponent $\gamma$ is not valid.

(ii) The streched exponential is not valid for very small $\gamma=0.01$, i.e. near $1 / f$.

\subsection{Potential predictability of extreme event return times}

For time series with weak LTM (correlation exponents $\gamma=0.4,0.7$, Bunde et al., 2004) the sequences $t_{r}(n)$ composed of extreme event return times show long-term correlations with similar LTM as the time series itself. To analyse this behavior in the vicinity of $1 / f$ noise, the correlation exponent is $\gamma=0.01$ (as in Sect. 4.1) and the extreme events are based on different thresholds providing the mean return times $R_{q}=10,100,1000,10000$. The total length of the time series is $N=10^{8}$. Note that this is two decades longer than $N=2^{21} \approx 2.1 \cdot 10^{6}$ in Eichner et al. (2007). The sequence of the return times is analyzed by detrended fluctuation analysis (DFA). Figure 6 shows that the long-term correlation of the return times is described by a power-law fluctuation function $F(n) \sim n^{\alpha}$ with $\alpha \approx 0.72$ independent of the threshold and the mean return time; the index $n$ enumerates the return times. This corresponds to the power spectrum exponent $\beta \approx 0.44$ (using $\beta=2 \alpha-1$ ) and the correlation exponent $\gamma \approx 0.56$. Thus the sequence of extreme events in time series near the $1 / f$-limit shows distinctly weaker long-term correlation properties than the original time series. 


\section{Conclusions}

This paper presents an analysis of the extreme event return time statistics for observed and simulated data with $1 / f$ power spectra. The observed data is given by measurements of temperature and mixing ratio during TOGACOARE (November 1992-February 1993) at the research vessel Kexue. In the time series of one minute resolution, 61 days with low numbers of missing values are extracted. Both time series show a scaling power spectrum, $S(f) \sim f^{-\beta}$, with $\beta=1 \ldots 1.2$; the correlation exponent in $C(t) \sim t^{-\gamma}$ is related by $\gamma=1-\beta$. This result is determined by detrended fluctuation analysis and substantiated by a fit of a FARIMA $(p, d, 0)$ fractionally differenced autoregressive process which yields $d \approx 0.5$ for the long-term behavior $(\beta=2 d)$. Hence, both time series are considered as $1 / f$ noise.

Extreme events are determined by Peak-Over-Threshold (POT) crossing. The observed return time distributions $p_{q}\left(t_{r}\right)$ are compared to a stretched exponential, $\sim \exp \left(-t^{\gamma}\right)$, and a Weibull distribution, $\sim t^{\gamma-1} \exp \left(-t^{\gamma}\right)$. According to the approach by Altmann and Kantz (2005) and Eichner et al. (2007), the stretched exponential distribution converges to a power-law $p_{q}\left(t_{r}\right) \sim t_{r}^{-s}$ with $s=1.5$ for $\gamma \rightarrow 0$.

The return time distributions for the two observed time series are better approximated by a Weibull distribution than by a power-law. If a power-law is fitted in the intermediate range of return times, the temperature yields a power-law exponent $s=1.74$, while the mixing ratio yields $s=1.8$; both are distinctly different from the stretched exponential limit.

Simulated data is generated by a fractionally differenced autoregressive process with a power spectrum in the vicinity of the stationarity threshold, $\beta=0.99(\gamma=0.01)$. As for the observational data, the Weibull distribution yields a convincing representation of the return time distributions, while a power-law can be excluded.

The simulated data is used to evaluate the potential predictability of the extreme event return times. The LTM in the sequence of return times is analyzed by the detrended fluctuation analysis and reveals a power law fluctuation function $F(n) \sim n^{\alpha}$ with $\alpha \approx 0.72$ (correlation exponent $\bar{\gamma} \approx 0.56$ ). Thus, the return time sequences show weaker long-term correlations than the original time series. This values is independent of the threshold and the mean return time. A possible reason is that short term random effects lead to level crossings which, thereby, perturb the overall LTM of the original time series.

The analysis leads to the following main conclusions for the behavior of extreme event return times in the $1 / f$ limit:

(i) The return time distributions for time series in the vicinity of a $1 / f$ power spectum are well approximated by the standard Weibull distribution. This is suggested by the observed time series and substantiated by the simulated data. The stretched exponential (which differs from Weibull by the absence of a prefactor) is likely to be convenient for weak LTM.

(ii) The sequence of return times shows LTM with $F(n) \sim n^{\alpha}$ with $\alpha \approx 0.72$ which is weaker than in the original time series $(\alpha=0.995)$. However, the correlation $C(n) \sim n^{-0.56}$ is still promising for the prediction of return times.

Future analyses should consider the Weibull distribution as an alternative to the stretched exponential return time distribution for a wide range of LTM correlation coefficients $\gamma$.

Acknowledgements. We like to thank the reviewers and the editor for their useful comments. This work has been supported by the EC project E2C2, Contract No. 012975.

Edited by: B. D. Malamud

Reviewed by: H. Rust and three other anonymous referees

\section{References}

Abaimov, S. G., Turcotte, D. L., Shcherbakov, R., and Rundle, J. B.,: Recurrence and interoccurrence behavior of self-organized complex phenomena, Nonlin. Processes Geophys., 14, 455-464, 2007, http://www.nonlin-processes-geophys.net/14/455/2007/.

Altmann, E. G. and Kantz, H.: Recurrence time analysis, long-term correlations, and extreme events, Phys. Rev. E, 71, 056106, 2005.

Beran, J.: Statistics for long-memory processes, Chapman \& Hall/CRC Press, London, 315 pp., 1994.

Blender, R. and Fraedrich, K.: Long time memory in global warming simulations, Geophys. Res. Lett., 30(14), 1769-1772, 2003.

Bunde, A., Eichner, J. F., Havlin, S., and Kantelhardt, J. W.: Return intervals of rare events in records with long-term persistence, Physica A, 342, 308-314, 2004.

Bunde, A., Eichner, J. F., Kantelhardt, J. W., and Havlin, S.: Longterm memory: A natural mechanism for the clustering of extreme events and anomalous residual times in climate records, Phys. Rev. Lett., 94, 048701, 2005.

Clauset, A., Shalizi, C. R., and Newman, M. E. J.: Power-law distributions in empirical data, http://arxiv.org/abs/0706.1062, 2007.

Corral, A.: Long-term clustering, scaling, and universality in the temporal occurrence of earthquakes, Phys. Rev. Lett., 92, 108501, 2004.

Eichner, J. F., Kantelhardt, J. W., Bunde, A., and Havlin, S.: Statistics of return intervals in long-term correlated records, Phys. Rev. E, 75, 011128, 2007.

Fraedrich, K. and Blender, R.: Scaling of atmosphere and ocean temperature correlations in observations and climate models, Phys. Rev. Lett., 90, 108501, 2003.

Hosking, J. R. M.: Fractional differencing, Biometrika, 68, 165176, 1981.

Huybers, P. and Curry, W.: Links between annual, Milankovitch and continuum temperature variability, Nature, 441, 329-332, 2006.

Lucas, C. and Zipser, E. J.: Environmental variability during TOGA COARE, J. Atmos. Sci., 57, 2333-2350, 2000. 
Monetti, R. A., Havlin, S., and Bunde, A.: Long-term persistence in the sea surface temperature fluctuations, Physica A, 320, 581589, 2003.

Maraun, D., Rust, H. W., and Timmer, J.: Tempting long-term memory - on the interpretation of DFA results, Nonlin. Processes Geophys., 11, 495-503, 2004,

http://www.nonlin-processes-geophys.net/11/495/2004/.

Newell, G. F. and Rosenblatt, M.: Zero crossing probabilities for Gaussian stationary processes, Ann. Math. Stat. 33, 1306, 1962.

Olla, P.: Return times for stochastic processes with power-law scaling. Phys. Rev. E, 76, 011122, 2007.

Peng, C.-K., Buldyrev, S. V., Havlin, S., Simons, M., Stanley, H. E., and Goldberger, A. L.: On the mosaic organization of DNA sequences. Phys. Rev. E, 49, 1685-1689, 1994.

R Development Core Team, R: A language and environment for statistical computing, Vienna, Austria, http://www.R-project.org, 2005.
Sornette, D.: Critical phenomena in natural sciences, 2nd ed., Springer Series in Synergetics, Springer-Verlag, Heidelberg, 528 p., 2006.

Webster, P. and Lukas, R.: TOGA COARE: the Coupled OceanAtmosphere Response Experiment, Bull. Amer. Meteor. Soc., 73, 1377-1416, 1992.

Weissman, M. B.: $1 / f$ noise and other slow, nonexponential kinetics in condensed matter, Rev. Mod. Phys., 60, 537-571, 1988.

Yano, J.-I., Fraedrich, K., and Blender, R.: Tropical convective variability as 1/f-noise, Journal of Climate, 14, 3608-3616, 2001.

Yano, J.-I., Blender, R., Zhang, C., and Fraedrich, K.: 1/f-Noise and pulse-like events in the tropical atmospheric surface variabilities: Convective self-criticality and impacts on ENSO, Quart. J. Roy. Meteorol. Soc., 130, 1697-1721, 2004.

Zaslavsky, G. M.: Chaos, fractional kinetics, and anomalous transport, Physics Reports, 371, 461-580, 2002. 